\title{
CONVERSE SYMMETRY AND INTERMEDIATE ENERGY VALUES IN REARRANGEMENT OPTIMIZATION PROBLEMS*
}

\author{
YICHEN LIU ${ }^{\dagger}$ AND BEHROUZ EMAMIZADEH ${ }^{\ddagger}$
}

\begin{abstract}
This paper discusses three rearrangement optimization problems where the energy functional is connected with the Dirichlet or Robin boundary value problems. First, we consider a simple model of Dirichlet type, derive a symmetry result, and prove an intermediate energy theorem. For this model, we show that if the optimal domain (or its complement) is a ball centered at the origin, then the original domain must be a ball. As for the intermediate energy theorem, we show that if $\alpha, \beta$ denote the optimal values of corresponding minimization and maximization problems, respectively, then every $\gamma$ in $(\alpha, \beta)$ is achieved by solving a max-min problem. Second, we investigate a similar symmetry problem for the Dirichlet problems where the energy functional is nonlinear. Finally, we show the existence and uniqueness of rearrangement minimization problems associated with the Robin problems. In addition, we shall obtain a symmetry and a related asymptotic result.
\end{abstract}

Key words. rearrangements, optimal solutions, symmetry, energy values, Robin problems, asymptotic

AMS subject classifications. 49A20, 49A40, 49N99, 35J20

DOI. $10.1137 / 16 \mathrm{M} 1100307$

1. Introduction. A rearrangement optimization problem is an optimization problem of the following forms:

$$
\inf _{f \in \mathcal{R}\left(f_{0}\right)} \Phi(f) \text { and } \sup _{f \in \mathcal{R}\left(f_{0}\right)} \Phi(f),
$$

where $\mathcal{R}\left(f_{0}\right)$ is a rearrangement class $^{1}$ generated by $f_{0}$, a prescribed function. The goal function $\Phi$ is frequently a nonlinear functional which arises from a boundary value problem. Burton in his celebrated papers [7, 8] established a well-developed theory of rearrangements which can be used to investigate problems like (1.1). Let us give an example. In $[7,8,9]$, the authors considered the functional

$$
\Phi(f) \equiv \int_{D} f u_{f} d x
$$

where $u_{f}$ is the unique solution of the classical Poisson's problem

$$
\begin{cases}-\Delta u=f & \text { in } D \\ u=0 & \text { on } \partial D .\end{cases}
$$

They proved the minimization and maximization problems (1.1) are both solvable. Given that the problem (1.3) physically describes the steady state of an elastic membrane, fixed at the boundary, subject to a vertical force $f(x)$, the functional (1.2) measures the total displacement of the membrane from the rest position. In this

\footnotetext{
* Received by the editors October 26, 2016; accepted for publication (in revised form) April 20, 2017; published electronically June 27, 2017.

http://www.siam.org/journals/sicon/55-3/M110030.html

${ }^{\dagger}$ Beijing International Center for Mathematical Research, Peking University, Beijing 100871, China (yichen.liu07@yahoo.com).

${ }_{\ddagger}^{\ddagger}$ School of Mathematical Sciences, The University of Nottingham-Ningbo, Ningbo 31510, China (Behrouz.Emamizadeh@nottingham.edu.cn).

${ }^{1}$ See Definition 2.2 .
} 
physical setting, the function $u_{f}$ denotes the displacement, whence, the optimization problems (1.1) address the question of finding the level of vulnerability of the membrane relative to rearranged force in $\mathcal{R}\left(f_{0}\right)$. Motivated by the abovementioned papers, other authors (see, for example, $[10,15,25]$ ), studied the same problems (1.1) except that $u_{f}$ is the solution of the following boundary value problem

$$
\begin{cases}-\Delta_{p} u=f & \text { in } D \\ u=0 & \text { on } \partial D\end{cases}
$$

where $\Delta_{p}$ is the classical $p$-Laplace operator, i.e., $\Delta_{p} u=\nabla \cdot\left(|\nabla u|^{p-2} \nabla u\right)$ with $1<$ $p<\infty$. In addition to presenting technical challenges, the results achieved in the corresponding optimization problems are physically significant because the $p$-Laplace operator is an operator frequently used to model physical phenomena occurring, for example, in non-Newtonian fluids, nonlinear elasticity, and glaciology; see [1, 2, 13].

Before stating the main results of our paper, we make some preparations. Let us return to the minimization and maximization problems (1.1), and denote $\alpha=$ $\inf _{f \in R\left(f_{0}\right)} \Phi(f), \beta=\sup _{f \in R\left(f_{0}\right)} \Phi(f)$. Quite often, the main concern arises when neither $\alpha$ nor $\beta$ is attained; see, for example, [25, Theorem 3.1] or [24, Theorem 3.6]. In such a situation, a natural remedy is to look for a rearrangement of $f_{0}$, denoted $\tilde{f}$, for which $\gamma=\Phi(\tilde{f})$ is close to our desired value $\alpha$ or $\beta$. So, the natural question is whether it is possible to find $f \in \mathcal{R}\left(f_{0}\right)$ such that $\Phi(f)=\gamma$ for a given number $\gamma \in(\alpha, \beta)$. Although we will discuss a problem where $\alpha$ and $\beta$ are attained in this article, our intention is to present some ideas to attack such problems about intermediate energy values. This is one of the features of our paper; see section 3.

Another feature is a symmetry result. In many rearrangement optimization problems, quite often, the optimal solutions inherit the same symmetry as the domain $D$. So, for example, if $D$ is Steiner symmetric, the optimal solutions turn out to be Steiner symmetric as well. For the minimization and maximization (1.1) considered in the setting of (1.2) and (1.3), the authors (see [9]), proved in the case $D$ is radial, both the minimizer and maximizer are unique, and they are indeed radial. However, to the best of our knowledge, the converse of these problems has never be addressed. In this paper, we discuss the converse in a special case.

The last part of the paper is devoted to a rearrangement minimization problem associated with the following Robin boundary value problem:

$$
\begin{cases}-\Delta u=f & \text { in } D, \\ \frac{\partial u}{\partial \nu}+\beta u=0 & \text { on } \partial D\end{cases}
$$

where $\beta$ is a positive constant, and the force function $f(x)$ is nonnegative and square integrable. Specifically, we shall prove the minimization problem in (1.1), where $\Phi(f)=\int_{D} f u_{f} d x$ with $u_{f}$ denoting the solution of (1.4) has a unique solution. Furthermore, we show if $D$ is radial, the minimizer is radially nondecreasing. We also address the converse for a particular class of rearrangement, where the generator is a characteristic function. We conclude the paper with an asymptotic result. In this result, we shall show that as $\beta$ tends to infinity, the corresponding minimizer of the Robin problem converges to the minimizer of the Dirichlet problem in $L^{2}(D)$.

Remark 1.1. Rearrangement optimization problems have strong connections with shape optimization problems when the prescribed function $f_{0}$ is a characteristic function. Let $f_{0}=\chi_{E_{0}}$ for some $E_{0} \subseteq D$ with $0<\left|E_{0}\right|<|D|$. The rearrangement optimization problems (1.1) can be reformulated as the following shape optimization 
problems:

$$
\inf _{|E|=\left|E_{0}\right|, E \subseteq D} \Psi(E):=\Phi\left(\chi_{E}\right) \text { and } \sup _{|E|=\left|E_{0}\right|, E \subseteq D} \Psi(E)
$$

For the discussions about such problems, we refer to $[11,20]$. In particular, the readers may consult [19] for symmetry problems arising in shape optimization.

Structure of the paper. In section 2 we state some well-known results about rearrangement of functions and spherical symmetrizations. Section 3 is devoted to a simple rearrangement optimization problem where the goal functional $\Phi_{s}$ is linear. In section 4, we shall prove a symmetry result regarding (1.1) where the functional $\Phi(f)$ is associated with the Dirichlet problem (1.3). The final section investigates the minimization problem in (1.1) related to the Robin problem (1.4), and concludes with the asymptotic result mentioned above.

2. Preliminaries. First, we will review some basic results about rearrangement theory from $[7,8]$. Let us start with the following definitions.

Definition 2.1. Let $D \subseteq \mathbb{R}^{N}$ and $D^{\prime} \subseteq \mathbb{R}^{M}$ be measurable with $|D|=\left|D^{\prime}\right|$. Suppose $f: D \rightarrow \mathbb{R}$ and $g: D^{\prime} \rightarrow \mathbb{R}$ are measurable functions. We say $f$ is a rearrangement of $g$ if and only if

$$
\lambda_{f}(\alpha):=|\{x \in D: f(x) \geq \alpha\}|=\left|\left\{x \in D^{\prime}: g(x) \geq \alpha\right\}\right|=: \lambda_{g}(\alpha) \quad \forall \alpha \in \mathbb{R} .
$$

Definition 2.2. Let $D, D^{\prime}$, and $g$ be as in Definition 2.1. The rearrangement class generated by $g$ on $D$, denoted $\mathcal{R}_{D}(g)$, is defined as

$$
\mathcal{R}_{D}(g):=\{f: D \rightarrow \mathbb{R} \text { measurable }: f \text { is a rearrangement of } g\} .
$$

Furthermore, we will set $\mathcal{R}(g) \equiv \mathcal{R}_{D}(g)$ if the rearrangement class is generated on $D$.

Definition 2.3. Let $f: D \rightarrow \mathbb{R}$ be measurable. The decreasing rearrangement of $f$ on $(0,|D|)$ is defined by $f^{\Delta}(s):=\max \left\{\alpha: \lambda_{f}(\alpha) \geq s\right\}$. Also, the increasing rearrangement of $f$ is defined by $f_{\Delta}(s):=f^{\Delta}(|D|-s)$. It is clear that $f^{\Delta}$ and $f_{\Delta}$ are rearrangements of $f$; see $[7$, Lemma 1$]$.

Definition 2.4. Let $f: D \rightarrow \mathbb{R}$ be a measurable function and $S$ be a measurable subset of $D$. We say the graph of $f$ has no significant flat sections on $S$ if and only if

$$
|\{x \in S: f(x)=\alpha\}|=0 \quad \forall \alpha \in \mathbb{R} .
$$

We recall a basic property of $f^{\Delta}$ here.

Lemma 2.5. $f^{\Delta}(\cdot)$ is left continuous on $(0,|D|)$.

The following lemmas related to the rearrangement class are vital in our analysis.

Lemma 2.6. Let $f \in L^{2}(D)$. Recalling that $\mathcal{R}(f)$ is the rearrangement class generated by $f$ on $D$, we have

(i) $\mathcal{R}(f) \subseteq L^{2}(D), \int_{D} f d x=\int_{D} g d x$, and $\|f\|_{2}=\|g\|_{2}$ for all $g \in \mathcal{R}(f)$;

(ii) $\overline{\mathcal{R}(f)}$, the weak closure of $\mathcal{R}(f)$ in $L^{2}(D)$, is convex and weakly sequentially compact. Moreover, it is metrizable with respect to the weak topology in $L^{2}(D)$

(iii) $\overline{\mathcal{R}(f)}=\left\{g \in L^{1}(D): \int_{D} f d x=\int_{D} g d x, \int_{0}^{s} g^{\Delta} d t \leq \int_{0}^{s} f^{\Delta} d t \forall s \in(0,|D|)\right\}$.

\footnotetext{
${ }^{2}$ We mean Lebesgue measurable in this article.
}

Copyright (C) by SIAM. Unauthorized reproduction of this article is prohibited. 
Proof. For the proof see [8, Lemmas 2.1, 2.2, and 2.3].

Lemma 2.7. Let $f, g: D \rightarrow \mathbb{R}$ be measurable functions. Suppose the graph of $g$ has no significant flat sections throughout $D$. Then, there exists a nonincreasing (or nondecreasing) function $\eta$ such that $\eta(g)$ is a rearrangement of $f$.

Proof. For the proof see [15, Lemma 2.2] or [8, Lemma 2.9].

Lemma 2.8. Let $f, g \in L^{2}(D)$. If there is a nonincreasing (or nondecreasing) function $\eta$ such that $\eta(g) \in \mathcal{R}(f)$, then $\eta(g)$ is the unique minimizer (or maximizer) of the linear functional $L(h)=\int_{D} h g d x$ relative to $h \in \overline{\mathcal{R}(f)}$. Moreover, we have $L(\eta \circ g)=\int_{0}^{|D|} f_{\Delta} g^{\Delta} d t\left(\right.$ or $\left.L(\eta \circ g)=\int_{0}^{|D|} f^{\Delta} g^{\Delta} d t\right)$.

Proof. For the proof see [8, Lemma 2.4 (ii)].

We need the following two basic lemmas.

Lemma 2.9. Suppose $\left\{f_{n}\right\} \subseteq L_{+}^{2}(D)$, and $f \in L^{2}(D)$. Suppose $f_{n} \rightarrow f$ in $L^{2}(D)$. Then, $f$ is nonnegative a.e. in $D$.

Proof. This is an immediate consequence of the Mazur lemma ([4, Corollary 3.8]), and the details are left to the reader.

The following is a variant of [7, Lemma 2].

Lemma 2.10. Suppose $f: D \rightarrow[0, \infty)$ is measurable, then

$$
\int_{E} f d x \geq \int_{0}^{|E|} f_{\Delta}(s) d s
$$

for every measurable subset $E \subseteq D$.

For $f \in L_{+}^{2}(D)$, we denote the support of $f$ on $D$ by $S(f)$, i.e., $S(f) \equiv\{f>0\} .{ }^{3}$ Because of Lemma 2.9, it is reasonable to introduce the following result.

Lemma 2.11. Let $f_{0} \in L_{+}^{2}(D)$. For every $f$ in $\overline{\mathcal{R}\left(f_{0}\right)},\left|S\left(f_{0}\right)\right| \leq|S(f)|$.

Proof. In order to derive a contradiction, let us assume $|S(f)|<\left|S\left(f_{0}\right)\right|$. Hence, $\alpha \equiv \int_{0}^{\left|S(f)^{c}\right|} f_{0_{\Delta}} d x$ is positive. Since $f \in \overline{\mathcal{R}\left(f_{0}\right)}$, there exists $\left\{f_{n}\right\} \subseteq \mathcal{R}\left(f_{0}\right)$ such that $f_{n} \rightarrow f$ in $L^{2}(D)$. Then we have

$$
\begin{aligned}
\alpha=\int_{0}^{\left|S(f)^{c}\right|} f_{0_{\Delta}} d x=\int_{0}^{\left|S(f)^{c}\right|} f_{n_{\Delta}} d x & \leq \int_{S(f)^{c}} f_{n} d x=\int_{D} f_{n} \chi_{S(f)^{c}} d x \\
& \rightarrow \int_{D} f \chi_{S(f)^{c}} d x=\int_{S(f)^{c}} f d x=0,
\end{aligned}
$$

which is a contradiction. The inequality in (2.1) is a consequence of Lemma 2.10.

Then, we introduce a technical lemma which will be useful in section 5 .

Lemma 2.12. Let $f_{0} \in L_{+}^{2}(D)$ and $g \in L^{2}(D)$. Let $\hat{f}$ be a minimizer of the linear functional $L(h)=\int_{D} h g d x$ relative to $h \in \overline{\mathcal{R}\left(f_{0}\right)}$. Suppose the graph of $g$ has no significant flat sections on $S(\hat{f})$. Then, there exists a nonincreasing $\eta$ such that $\eta(g)$ is a rearrangement of $f_{0}$.

Proof. From Lemma 2.11, we know there exists $f_{1} \in \mathcal{R}\left(f_{0}\right)$ such that $S\left(f_{1}\right) \subseteq$ $S(\hat{f})$. Since the graph of $g$ has no significant flat sections on $S(\hat{f})$, by using Lemma 2.7

\footnotetext{
${ }^{3}$ We set $\{f>0\} \equiv\{x \in D: f(x)>0\}$ for brevity, and similarly for other situations.
}

Copyright $@$ by SIAM. Unauthorized reproduction of this article is prohibited. 
we infer the existence of nonincreasing $\eta_{S}$ such that $\eta_{S}\left(\left.g\right|_{S(\hat{f})}\right)$ is a rearrangement of $\left.f_{1}\right|_{S(\hat{f})}$. In order to extend $\eta_{S}$ to $\eta$ so that $\eta(g) \in \mathcal{R}\left(f_{0}\right)$, we intend to show

$$
\underset{S(\hat{f})^{c}}{\operatorname{essinf}} g \geq \underset{S(\hat{f})}{\operatorname{ess} \sup } g \equiv \gamma .
$$

To prove (2.2), we argue by contradiction and suppose there exists $A \subseteq S(\hat{f})^{c}, B \subseteq$ $S(\hat{f})$, and $\alpha>\beta$ such that $g \geq \alpha$ in $B$ and $g \leq \beta$ in $A$. Without loss of generality, we may assume $|A|=|B|$, otherwise, we could choose subsets instead. Let $\rho: A \rightarrow B$ be a measure preserving bijection: such a map exists; see [26]. At this stage, let us define $\tilde{f}: D \rightarrow \mathbb{R}$ by

$$
\tilde{f}(x)= \begin{cases}\hat{f}(x) & x \in(A \cup B)^{c}, \\ \hat{f}(\rho(x)) & x \in A, \\ \hat{f}\left(\rho^{-1}(x)\right) & x \in B .\end{cases}
$$

Observing that $\tilde{f} \in \overline{\mathcal{R}\left(f_{0}\right)}$ by Lemma $2.6(\mathrm{i})$ and (iii), we compute

$$
\begin{aligned}
\int_{D} \tilde{f} g d x-\int_{D} \hat{f} g d x & =\int_{A \cup B} \tilde{f} g d x-\int_{A \cup B} \hat{f} g d x=\int_{A} \tilde{f} g d x-\int_{B} \hat{f} g d x \\
& =\int_{A}(\hat{f} \circ \rho) g d x-\int_{B} \hat{f} g d x=\int_{B} \hat{f}\left(g \circ \rho^{-1}\right) d x-\int_{B} \hat{f} g d x \\
& \leq(\beta-\alpha) \int_{B} \hat{f} d x<0,
\end{aligned}
$$

which contradicts the minimality of $\hat{f}$. Therefore, we have shown (2.2). To finish the proof, we construct $\eta$ as follows:

$$
\eta(t)= \begin{cases}\eta_{S}(t) & t<\gamma \\ 0 & t \geq \gamma\end{cases}
$$

Obviously, $\eta$ is nonincreasing and $\eta(g) \in \mathcal{R}\left(f_{1}\right)=\mathcal{R}\left(f_{0}\right)$ as desired.

Next, we give a brief exposition of spherical symmetrization; see [5, 12, 27] for details. ${ }^{4}$ Given a measurable set $K \subseteq \mathbb{R}^{N}$, we fix a unit vector $\vec{e}$ as the direction. Then, the spherical symmetrization of $K$ with respect to direction $\vec{e}$, denoted by $K^{*}$, is characterized by the following property: for every $r \in(0, \infty)$, the set $K^{*} \cap \partial B(0, r)$ is a spherical cap centered at $r \vec{e}$ satisfying

$$
\mathcal{H}^{N-1}\left(K^{*} \cap \partial B(0, r)\right)=\mathcal{H}^{N-1}(K \cap \partial B(0, r)) \quad \forall 0<r<\infty,
$$

where $\mathcal{H}^{N-1}$ denotes the $(N-1)$-dimensional Hausdorff measure in $\mathbb{R}^{N}$, and $B(0, r)$ is an open ball centered at the origin with radius $r$. For a measurable function $u$, the spherical symmetrization $u^{*}$ is constructed such that

$$
\left\{u^{*} \geq t\right\}=\{u \geq t\}^{*} \quad \forall t \in \mathbb{R} .
$$

We will need the following well-known results about spherical symmetrization.

Lemma 2.13. Let $D$ be a ball centered at the origin and $u \in H^{1}(D)$. Then,

\footnotetext{
${ }^{4}$ In some books or papers, spherical symmetrization is also called cap symmetrization.
} 
(i) $u^{*} \in H^{1}(D)$;

(ii) if $f: \mathbb{R} \rightarrow \mathbb{R}$ is continuous and $f(u) \in L^{1}(D)$ (or $f(u) \in L^{1}(\partial D)$ ), then we have $\int_{D} f(u) d x=\int_{D} f\left(u^{*}\right) d x\left(\right.$ or $\left.\int_{\partial D} f(u) d \mathcal{H}^{N-1}=\int_{\partial D} f\left(u^{*}\right) d \mathcal{H}^{N-1}\right)$;

(iii) $\int_{D}\left|\nabla u^{*}\right|^{2} d x \leq \int_{D}|\nabla u|^{2} d x$.

Proof. For (i) and (iii), see [5, Theorem 4.5]. For (ii), see [5, Lemma 3.3] or [8, Lemma 2.1].

Finally, we recall a special case of the Radon-Riesz theorem.

Lemma 2.14. Let $D \subseteq \mathbb{R}^{N}$ be measurable, $f \in L^{2}(D)$, and $\left\{f_{n}\right\} \subseteq L^{2}(D)$. If $f_{n} \rightarrow f$ in $L^{2}(D)$ and $\lim _{n \rightarrow \infty}\left\|f_{n}\right\|_{2}=\|f\|_{2}$, then $f_{n} \rightarrow f$ in $L^{2}(D)$.

3. Simple model. Consider the boundary value problem

$$
\begin{cases}-\Delta u=f & \text { in } D \\ u=0 & \text { on } \partial D\end{cases}
$$

where $D$ is a bounded domain in $\mathbb{R}^{N}(N \geq 2)$ with Lipschitz boundary. For each $f \in L^{2}(D)$, we denote the unique solution of $(3.1)$ by $u_{f} \in H_{0}^{1}(D)$. Let us fix a nontrivial, nonnegative $f_{0} \in L^{2}(D)$. We are interested in the following rearrangement optimization problems:

$$
\inf _{f \in \mathcal{R}\left(f_{0}\right)} \Phi_{s}(f):=\int_{D} u_{f} d x
$$

and

$$
\sup _{f \in \mathcal{R}\left(f_{0}\right)} \Phi_{s}(f) .
$$

Before stating the first main result, we introduce the Saint-Venant problem:

$$
\begin{cases}-\Delta w=1 & \text { in } D \\ w=0 & \text { on } \partial D .\end{cases}
$$

THEOREM 3.1. The problem (3.2) (or (3.3)) has a unique solution $\hat{f} \in \mathcal{R}\left(f_{0}\right)$. Moreover, we have $\hat{f}=\eta(w)$ a.e. for some nonincreasing (or nondecreasing) $\eta$.

To prove Theorem 3.1, we first observe that $\Phi_{s}(f)=\int_{D} f w d x$. This follows immediately from the divergence theorem.

Proof of Theorem 3.1. We prove (3.2) is solvable and leave the solvability of the proof of (3.3) to the reader.

As mentioned above, the problem (3.2) can be formulated as follows:

$$
\inf _{f \in \mathcal{R}\left(f_{0}\right)} \int_{D} f w d x .
$$

Since the graph of $w$ has no significant flat sections on $D$ by [18, Lemma 7.7], in conjunction with Lemma 2.7, we infer the existence of a nonincreasing $\eta$ such that $\eta(w)$ is a rearrangement of $f_{0}$. Applying Lemma 2.8, we deduce $\eta(w)$ is the unique minimizer of (3.5). This completes the proof of the theorem.

Corollary 3.2. Let $E$ be a measurable subset of $D$ satisfying $0<|E|<|D|$, and let $f_{0}=\chi_{E}$. The problem (3.2) (or (3.3)) has a unique solution $\chi_{\hat{E}} \in \mathcal{R}\left(\chi_{E}\right)$, 
where ${ }^{5} \hat{E}=\{w<m\}$ (or $\hat{E}=\{w>m\}$ ) for some $m>0$. If $D$ is a ball centered at the origin with radius a, then $\hat{E}^{c}$ (or $\hat{E}$ ) is a ball centered at the origin.

Proof. Observe that $w(x)=-\frac{|x|^{2}}{2 N}+\frac{a^{2}}{2 N}$, which is (strictly) radially decreasing, whence, the assertions readily follow from Theorem 3.1.

The second main result of this section is a converse of Corollary 3.2.

Theorem 3.3. Let $D$ be a bounded, connected domain in $\mathbb{R}^{N}(N \geq 2)$ with Lipschitz boundary, and consider a measurable set $E \subseteq D$ satisfying $0<|E|<|D|$. If the solution of (3.2) (or (3.3)) with $f_{0}=\chi_{E}$ is $\chi_{\hat{E}}$ in which $\hat{E}=\{w<m\}$ (or $\hat{E}=\{w>m\})$ for some $m>0$ and $\hat{E}^{c}$ (or $\hat{E}$ ) is a ball centered at the origin, then $D$ is also a ball concentric with $\hat{E}^{c}$ (or $\hat{E}$ ).

Proof. We only consider the minimization problem (3.2) with $f_{0}=\chi_{E}$, and the case of the maximization problem (3.3) can be treated similarly. Let us denote $R=$ $\sqrt[n]{\frac{|D|-|E|}{\omega_{N}}}$, where $\omega_{N}$ is the volume of a unit ball in $\mathbb{R}^{N}$, and let

$$
v(x)=-\frac{x^{2}}{2 N}+\frac{R^{2}}{2 N}+m \quad \forall x \in \bar{D} .
$$

By setting $z(x)=w(x)-v(x)$ for all $x \in \bar{D}$, we infer $z \in H^{1}(D)$ is a weak solution of

$$
\Delta z=0 \text { in } D .
$$

It is well known that $z$ is real analytic in $D$. On the other hand, $v$ satisfies

$$
\begin{cases}-\Delta v=1 & \text { in } \hat{E}^{c} \\ v=m & \text { on } \partial \hat{E}^{c} .\end{cases}
$$

Since $\hat{E}=\{w<m\}$, we infer $w$ also satisfies (3.6). By uniqueness, we must have $v=w$ on $\overline{\hat{E}^{c}}$, i.e., $z=0$ on $\overline{\hat{E}^{c}}$. Recalling that $z$ is real analytic in $D$, it results from $[3$, Theorem 1.27] that $z=0$ in $D$. Moreover, $w$ is radially decreasing in $\bar{D}$ since $v$ is. By the strong maximum principle (see [17, Theorem 2.13]), we have $w$ is (strictly) positive throughout $D$. As $w=0$ on $\partial D$, we must then have $D$ is a ball centered at the origin.

The next main result is the so called intermediate energy value theorem.

TheOREM 3.4. Let $D$ be a bounded domain in $\mathbb{R}^{N}(N \geq 2)$ with Lipschitz boundary, and consider $f_{0} \in L_{+}^{2}(D)$. Let $k$ and $K$ be the optimal values of the rearrangement optimization problems (3.2) and (3.3) respectively (see Theorem 3.1). Then, for all $\alpha$ in $(k, K)$, there exists a unique $\tilde{f} \in \mathcal{R}\left(f_{0}\right)$ such that $\Phi_{s}(\tilde{f})=\alpha$ and it verifies

$$
\Phi_{s}(\tilde{f})=\sup _{g \in \mathcal{R}_{1}(\gamma)} \inf _{h \in \mathcal{R}_{2}(\gamma)} \Phi_{s}(g+h)
$$

for some $\gamma \in(0,|D|)$, where $\mathcal{R}_{1}(\gamma) \equiv \mathcal{R}_{D}\left(f_{0}^{\Delta} \chi_{[0, \gamma]}\right)$ and $\mathcal{R}_{2}(\gamma) \equiv \mathcal{R}_{D}\left(f_{0}^{\Delta} \chi_{[\gamma,|D|]}\right)$. Moreover, there exist a positive constant $m_{\gamma}$, a nondecreasing function $\eta_{1}$, and a nonincreasing function $\eta_{2}$ such that

$$
\tilde{f}(x)= \begin{cases}\eta_{1}(w(x)), & x \in E_{\gamma}^{+} \equiv\left\{w>m_{\gamma}\right\}, \\ \eta_{2}(w(x)), & x \in E_{\gamma}^{-} \equiv\left\{w<m_{\gamma}\right\} .\end{cases}
$$

\footnotetext{
${ }^{5}$ For two measurable sets $A, B$ such that $A=B$, we mean $|A \Delta B|=0$, where $\Delta$ denotes the symmetric difference between two sets.
}

Copyright $@$ by SIAM. Unauthorized reproduction of this article is prohibited. 
Proof. Observe that

$$
\begin{aligned}
\sigma(\gamma) & :=\sup _{g \in \mathcal{R}_{1}(\gamma)} \inf _{h \in \mathcal{R}_{2}(\gamma)} \Phi_{s}(g+h)=\sup _{g \in \mathcal{R}_{1}(\gamma)} \inf _{h \in \mathcal{R}_{2}(\gamma)} \int_{D}(g+h) w d x \\
& =\sup _{g \in \mathcal{R}_{1}(\gamma)} \int_{D} g w d x+\inf _{h \in \mathcal{R}_{2}(\gamma)} \int_{D} h w d x \\
& =\int_{0}^{\gamma} f_{0}^{\Delta}(t) w^{\Delta}(t) d t+\int_{\gamma}^{|D|} f_{0}^{\Delta}(t) w^{\Delta}(\gamma+|D|-t) d t,
\end{aligned}
$$

where we have used the Hardy-Littlewood inequality in the last equality; see [7, Theorem 1], for example. Let us set $\beta=\left|S\left(f_{0}\right)\right|$. It is easily seen that $\sigma(0)=k$, and $\sigma(\gamma)=K$ for all $\gamma \in[\beta,|D|]$. We shall show that $\sigma(\cdot)$ is continuous on $[0, \beta]$. To this end, we extend the domain of $w^{\Delta}$ to $[0, \infty)$ by setting: $w^{\Delta}(0)=\sup _{D} w=\max _{\bar{D}} w$ and $w^{\Delta}(t)=0$ for all $t \geq|D|$. Let us fix $\gamma_{1}, \gamma_{2} \in[0, \beta]$, then we obtain

$$
\begin{aligned}
\sigma\left(\gamma_{2}\right)-\sigma\left(\gamma_{1}\right)= & \int_{\gamma_{1}}^{\gamma_{2}} f_{0}^{\Delta}(t) w^{\Delta}(t) d t \\
& -\int_{\gamma_{1}}^{|D|} f_{0}^{\Delta}(t)\left[w^{\Delta}\left(\gamma_{1}+|D|-t\right)-w^{\Delta}\left(\gamma_{2}+|D|-t\right)\right] d t \\
& -\int_{\gamma_{1}}^{\gamma_{2}} f_{0}^{\Delta}(t) w^{\Delta}\left(\gamma_{2}+|D|-t\right) d t
\end{aligned}
$$

Since the graph of $w$ has no significant flat sections on $D$, and $w$ is positive on $D, w^{\Delta}$ is (strictly) decreasing, and positive on $(0,|D|)$. From [28, Theorem 2], we infer $w$ is Lipschitz on $\bar{D}$. Applying the proof of [23, Proposition 4.2.4] or [6, Corollary 2.6], we have $w^{\Delta} \in C[0,|D|]$. This, in turn, implies $w^{\Delta}$ is uniformly continuous on $[0,|D|]$. From (3.10), it follows that $\sigma\left(\gamma_{1}\right) \rightarrow \sigma\left(\gamma_{2}\right)$ as $\gamma_{1} \rightarrow \gamma_{2}$. So, $\sigma(\cdot)$ is continuous on $[0,|D|]$. This result implies for $\alpha \in(k, K)$, there exists $\gamma \in(0, \beta)$ such that $\sigma(\gamma)=\alpha$. On the other hand, we can find $m_{\gamma}>0$ such that $\left|E_{\gamma}^{+}\right|=\gamma$ and $\left|E_{\gamma}^{-}\right|=|D|-\gamma$. From (3.9), we have

$$
\sigma(\gamma)=\sup _{g \in \mathcal{R}_{1}(\gamma)} \int_{E_{\gamma}^{+}} g w d x+\inf _{h \in \mathcal{R}_{2}(\gamma)} \int_{E_{\gamma}^{-}} h w d x .
$$

Since the graph of $w$ has no significant flat sections on $D$, we will obtain the representation in (3.8) from Lemmas 2.7 and 2.8. Furthermore, for every $\gamma \in(0,|D|)$, the solution to (3.7) is unique.

Finally, to prove the uniqueness of $\tilde{f}$, it suffices to show $\sigma(\cdot)$ is (strictly) increasing on $(0, \beta)$. To this end, we recall that $w$ is Lipschitz on $\bar{D}$. Hence, from the proof of [21, Theorem 2.3.2], we deduce $w^{\Delta}$ is Lipschitz on $(\epsilon,|D|]$ for any $0<\epsilon<|D|$. For $\gamma \in(0, \beta)$, applying Lemma 2.5, Proposition A.1, and Remark A.2 to (3.9), it yields

$$
\begin{aligned}
\sigma^{\prime}(\gamma-) & =-f_{0}^{\Delta}(\gamma) w^{\Delta}(\gamma)+f_{0}^{\Delta}(\gamma) w^{\Delta}(|D|)+\int_{\gamma}^{|D|} f_{0}^{\Delta}(t) \frac{\partial w^{\Delta}}{\partial \gamma-}(\gamma+|D|-t) d t \\
& =-f_{0}^{\Delta}(\gamma) w^{\Delta}(\gamma)+\int_{\gamma}^{\beta} f_{0}^{\Delta}(t) \frac{\partial w^{\Delta}}{\partial t}(\gamma+|D|-t) d t \\
& \leq-f_{0}^{\Delta}(\gamma) w^{\Delta}(\gamma)+f_{0}^{\Delta}(\gamma) \int_{\gamma}^{\beta} \frac{\partial w^{\Delta}}{\partial t}(\gamma+|D|-t) d t \\
& =f_{0}^{\Delta}(\gamma)\left[-w^{\Delta}(\gamma)+w^{\Delta}(\gamma+|D|-\beta)\right]<0,
\end{aligned}
$$

Copyright (c) by SIAM. Unauthorized reproduction of this article is prohibited. 
where we have used the fact that $w^{\Delta}$ is (strictly) decreasing on $[0,|D|]$ in the last inequality. Hence, it follows from Proposition A.3 that $\sigma(\cdot)$ is (strictly) increasing on $[0, \beta)$. This completes the proof of the theorem.

Corollary 3.5. Let $E, f_{0}$ be as in Corollary 3.2. Suppose $k$ and $K$ are the optimal values of the corresponding rearrangement optimization problems (3.2) and (3.3), respectively. Then, for all $\alpha$ in $(k, K)$, there exists a unique $\chi_{\hat{E}} \in \mathcal{R}\left(\chi_{E}\right)$ such that $\Phi_{s}\left(\chi_{\hat{E}}\right)=\alpha$ and it satisfies the max-min problem (3.7) for some $\gamma \in(0,|D|)$. Furthermore, $\hat{E}$ is comprised of two connected components: $\hat{E}_{1}=\left\{w>m_{1}\right\}$ for some $m_{1} \geq m_{\gamma}$ and $\hat{E}_{2}=\left\{w<m_{2}\right\}$ for some $m_{2} \leq m_{\gamma}$. If $D$ is a ball centered at the origin with radius a, then $\hat{E}_{1}$ is a ball centered at the origin, while $\hat{E}_{2}$ is a ring around the boundary.

Proof. The assertions follow from Theorem 3.4. In particular, the last assertion is due to the fact that $w$ is (strictly) radially decreasing.

The last result of this section is a converse of Corollary 3.5.

Theorem 3.6. Let $D, E$ be as in Theorem 3.3. Suppose $\alpha \in(k, K)$, and there exists $\chi_{\hat{E}} \in \mathcal{R}\left(\chi_{E}\right)$ such that $\Phi_{s}\left(\chi_{\hat{E}}\right)=\alpha$ and it satisfies the max-min problem (3.7) for some $\gamma \in(0,|D|)$. If the inner connected component of $\hat{E}$, denoted $\hat{E}_{1}$, is a ball centered at the origin and $\hat{E}_{1}=\{w>m\}$ for some $m>0$, then $D$ is also a ball centered at the origin.

Proof. The proof is a minor variant of the proof of Theorem 3.3.

4. Rearrangement optimization problems with Dirichlet boundary condition. In this section, we consider the same boundary value problem (3.1). As usual, $u_{f} \in H_{0}^{1}(D)$ is denoted to be the unique solution of (3.1) for $f \in L^{2}(D)$. We fix a nontrivial, nonnegative $f_{0} \in L^{2}(D)$. We are interested in the following rearrangement optimization problems:

$$
\inf _{f \in \mathcal{R}\left(f_{0}\right)} \Phi_{d}(f):=\int_{D} f u_{f} d x
$$

and

$$
\sup _{f \in \mathcal{R}\left(f_{0}\right)} \Phi_{d}(f) .
$$

We recall two known results here.

Proposition 4.1. The problem (4.1) (or (4.2)) has a solution $\hat{f} \in \mathcal{R}\left(f_{0}\right)$. Moreover, $\hat{f}=\eta\left(u_{\hat{f}}\right)$ a.e. in $D$ for some nonincreasing (or nondecreasing) function $\eta$. In particular, the minimizer for problem (4.1) is unique in $\mathcal{R}\left(f_{0}\right)$.

Proof. For the proof see [9, Theorem 2.1] and [7, Theorem 7].

Proposition 4.2. Let $D$ be a ball centered at the origin. Then, the problem (4.1) (or (4.2)) has a unique solution $\hat{f} \in \mathcal{R}\left(f_{0}\right)$. Moreover, $\hat{f}$ is radially nondecreasing (or nonincreasing).

Proof. For the proof see [9, Theorem 3.1].

Corollary 4.3. Let $E$ be a measurable subset of $D$ satisfying $0<|E|<|D|$, and let $f_{0}=\chi_{E}$. The problem (4.1) (or (4.2)) has a solution $\chi_{\hat{E}} \in \mathcal{R}\left(\chi_{E}\right)$, where $\hat{E}=\left\{u_{\chi_{\hat{E}}}<m\right\}$ (or $\hat{E}=\left\{u_{\chi_{\hat{E}}}>m\right\}$ ) for some $m>0$. If $D$ is a ball centered at 
TABLE 1

Test functions for the minimization problem (4.1).

\begin{tabular}{|c|c|}
\hline$N=2$ & $\begin{array}{cl}v(x)= \begin{cases}m, & x \in \hat{E}^{c}, \\
\frac{R^{2}-|x|^{2}}{4}+\frac{R^{2}}{2} \log \frac{|x|}{R}+m, & x \in \hat{E} \cup \partial D,\end{cases} \\
\text { where } R=\sqrt[n]{\frac{|D|-|E|}{\omega_{N}}} .\end{array}$ \\
\hline$N \geq 3$ & $\begin{array}{c}v(x)= \begin{cases}m, & x \in \hat{E}^{c}, \\
-\frac{|x|^{2}}{2 N}-\frac{R^{N}|x|^{2-N}}{N(N-2)}+\frac{R^{2}}{2(N-2)}+m, & x \in \hat{E} \cup \partial D,\end{cases} \\
\text { where } R=\sqrt[n]{\frac{|D|-|E|}{\omega_{N}} .}\end{array}$ \\
\hline
\end{tabular}

TABLE 2

Test functions for the maximization problem (4.2).

\begin{tabular}{|c|c|}
\hline$N=2$ & $v(x)=\left\{\begin{array}{ll}\frac{R^{2}-|x|^{2}}{4}+m, & x \in \hat{E}, \\
\frac{R^{2}}{2} \log \frac{R}{|x|}+m, & x \in \hat{E}^{c} \cup \partial D,\end{array} \quad\right.$ where $R=\sqrt[n]{\frac{|E|}{\omega_{N}}}$. \\
\hline$N \geq 3$ & $v(x)=\left\{\begin{array}{ll}\frac{R^{2}-|x|^{2}}{2 N}+m, & x \in \hat{E}, \\
\frac{R^{N}|x|^{2-N}}{N(N-2)}-\frac{R^{2}}{N(N-2)}+m, & x \in \hat{E}^{c} \cup \partial D,\end{array} \quad\right.$ where $R=\sqrt[n]{\frac{|E|}{\omega_{N}}}$. \\
\hline
\end{tabular}

the origin with radius a, then the minimizer (or maximizer) $\chi_{\hat{E}}$ is unique and $\hat{E}$ is a ring around the boundary (or a ball centered at the origin).

The main result of this section is the following theorem which is a converse of Corollary 4.3.

TheOREM 4.4. Let $D$ and $E$ be as in Theorem 3.3. If a solution of (4.1) (or (4.2)) with $f_{0}=\chi_{E}$ is $\chi_{\hat{E}}$ in which $\hat{E}=\left\{u_{\chi_{\hat{E}}}<m\right\}$ (or $\hat{E}=\left\{u_{\chi_{\hat{E}}}>m\right\}$ ) for some $m>0$ and $\hat{E}^{c}$ (or $\hat{E}$ ) is a ball centered at the origin, then $D$ is also a ball centered at the origin.

Proof. The proof is almost the same as the proof of Theorem 3.3, except that $u_{\chi_{\hat{E}}}$ plays the role of $w$ and different test functions, $v$, should be used. For the convenience of the readers, we list the test function for each case in Tables 1 and 2.

5. Rearrangement minimization problems with Robin boundary condition. Consider the boundary value problem

$$
\begin{cases}-\Delta u=f & \text { in } D, \\ \frac{\partial u}{\partial \nu}+\beta u=0 & \text { on } \partial D\end{cases}
$$

where $D$ is a bounded domain in $\mathbb{R}^{N}(N \geq 2)$ with $C^{1,1}$ boundary, $\nu$ denotes the outward unit normal vector on $\partial D$, and $\beta$ is a positive constant. For $f \in L^{2}(D)$, $u_{f} \in H^{1}(D)$ is a (weak) solution of (5.1) if and only if the following integral equation holds:

$$
\int_{D} \nabla u_{f} \cdot \nabla \varphi d x+\beta \int_{\partial D} u_{f} \varphi d \mathcal{H}^{N-1}=\int_{D} f \varphi d x \quad \forall \varphi \in H^{1}(D) .
$$

We denote the norm on $H^{1}(D)$ by $\|\cdot\|$, and we use $c$ or $C$ to denote constants that may vary from one step to another. 
It is classical that the boundary value problem (5.1) has a unique solution $u_{f} \in$ $H^{1}(D)$, which is the unique minimizer of the functional

$$
E(u):=\frac{1}{2} \int_{D}|\nabla u|^{2} d x+\frac{1}{2} \beta \int_{\partial D} u^{2} d \mathcal{H}^{N-1}-\int_{D} f u d x,
$$

relative to $u \in H^{1}(D)$. Moreover, the bilinear form $a: H^{1}(D) \times H^{1}(D) \rightarrow \mathbb{R}$ defined by

$$
a(u, v):=\int_{D} \nabla u \cdot \nabla v d x+\beta \int_{\partial D} u v d \mathcal{H}^{N-1} \quad \forall u, v \in H^{1}(D)
$$

is coercive, continuous, and symmetric.

We are interested in the following minimization problem:

$$
\inf _{f \in \mathcal{R}\left(f_{0}\right)} \Phi_{r}(f):=\int_{D} f u_{f} d x
$$

where $f_{0}$ is a nontrivial, nonnegative function in $L^{2}(D)$, and $u_{f}$ denotes the unique solution of (5.1) corresponding to $f$.

The first main result of this section is the following.

TheOREM 5.1. The problem (5.3) has a unique solution $\hat{f} \in \mathcal{R}\left(f_{0}\right)$. Moreover, we have $\hat{f}=\eta\left(u_{\hat{f}}\right)$ a.e. in $D$ for some nonincreasing function $\eta$.

To prove Theorem 5.1, we shall need the following.

LEMMA 5.2. The following statements are valid.

(i) $\Phi_{r}$ is weakly sequentially continuous on $L^{2}(D)$.

(ii) $\Phi_{r}$ is strictly convex.

(iii) $\Phi_{r}$ is Gâteaux differentiable. Moreover, the Gâteaux derivative of $\Phi_{r}$ at a given $f$, denoted $\Phi_{r}^{\prime}(f)$, can be identified with the function $2 u_{f}$.

Proof.

(i) Let $f_{n} \rightarrow f$ in $L^{2}(D)$ and set $u_{n} \equiv u_{f_{n}}$ for simplicity. By using (5.2) and recalling coercivity of $a(\cdot, \cdot)$, we have

$$
c\left\|u_{n}\right\|^{2} \leq a\left(u_{n}, u_{n}\right)=\int_{D} f_{n} u_{n} d x \leq\left\|f_{n}\right\|_{2}\left\|u_{n}\right\|_{2} \leq\left\|f_{n}\right\|_{2}\left\|u_{n}\right\| .
$$

So, $\left\{u_{n}\right\}$ is bounded in $H^{1}(D)$. Thus, we infer the existence of a subsequence, still denoted $\left\{u_{n}\right\}$, and $u \in H^{1}(D)$ such that $u_{n} \rightarrow u$ in $H^{1}(D), u_{n} \rightarrow u$ in $L^{2}(D)$, and $u_{n} \rightarrow u$ in $L^{2}(\partial D)$. From the definition, we find

$$
\int_{D} \nabla u_{n} \cdot \nabla \varphi d x+\beta \int_{\partial D} u_{n} \varphi d \mathcal{H}^{N-1}=\int_{D} f_{n} \varphi d x \quad \forall \varphi \in H^{1}(D) .
$$

Passing to limit in the above equation yields

$$
\int_{D} \nabla u \cdot \nabla \varphi d x+\beta \int_{\partial D} u \varphi d \mathcal{H}^{N-1}=\int_{D} f \varphi d x \quad \forall \varphi \in H^{1}(D) .
$$

This means $u$ is a solution of (5.1) corresponding to $f$. Hence, $u=u_{f}$. Recalling that $u_{n} \rightarrow u=u_{f}$ in $L^{2}(D)$, we deduce

$$
\Phi_{r}\left(f_{n}\right)=\int_{D} f_{n} u_{n} d x \rightarrow \int_{D} f u_{f} d x=\Phi_{r}(f),
$$

as desired. 
(ii) From the variational formulation of $u_{f}$, we obtain

$$
\Phi_{r}(f)=-2 E\left(u_{f}\right)=\psi_{u_{f}}(f)=\sup _{u \in H^{1}(D)} \psi_{u}(f),
$$

where $\psi_{u}(f):=2 \int_{D} f u d x-\int_{D}|\nabla u|^{2} d x-\beta \int_{\partial D} u^{2} d \mathcal{H}^{N-1}$ for all $u \in H^{1}(D)$. So, $\Phi_{r}$ is convex, being the supremum of a collection of affine functions. However, $\Phi_{r}$ is actually strictly convex. Indeed, we can prove this by the method of contradiction. To this end, suppose there exist $t \in(0,1), f, g \in$ $L^{2}(D)$, and $f \neq g$ such that

$$
\Phi_{r}(t f+(1-t) g)=t \Phi_{r}(f)+(1-t) \Phi_{r}(g) .
$$

Denoting $u_{t} \equiv u_{t f+(1-t) g}$, (5.5) leads to

$$
\begin{aligned}
2 \int_{D}[t f+ & (1-t) g] u_{t} d x-\int_{D}\left|\nabla u_{t}\right|^{2} d x-\beta \int_{\partial D} u_{t}^{2} d \mathcal{H}^{N-1} \\
= & t\left(2 \int_{D} f u_{f} d x-\int_{D}\left|\nabla u_{f}\right|^{2} d x-\beta \int_{\partial D} u_{f}^{2} d \mathcal{H}^{N-1}\right) \\
& +(1-t)\left(2 \int_{D} g u_{g} d x-\int_{D}\left|\nabla u_{g}\right|^{2} d x-\beta \int_{\partial D} u_{g}^{2} d \mathcal{H}^{N-1}\right) .
\end{aligned}
$$

By moving all terms to the right-hand side, we obtain

$$
t\left[\psi_{u_{f}}(f)-\psi_{u_{t}}(f)\right]+(1-t)\left[\psi_{u_{g}}(g)-\psi_{u_{t}}(g)\right]=0 .
$$

Recalling the supremum in (5.4) is uniquely attained, we must have $u_{f}=$ $u_{t}=u_{g}$ a.e. in $D$. Combined with (5.2), this clearly implies $f=g$ which is a contradiction.

(iii) Let us define the operator $K: L^{2}(D) \rightarrow H^{1}(D)$ by setting $K(f)=u_{f}$. It is easy to show $K$ is linear; furthermore, it is symmetric in the sense that

$$
\int_{D} f K g d x=\int_{D} g K f d x \quad \forall f, g \in L^{2}(D) .
$$

Fix $f, h \in L^{2}(D)$, we then have

$$
\Phi_{r}(f+t h)=\int_{D} f K f d x+2 t \int_{D} h K f d x+t^{2} \int_{D} h K h d x .
$$

Hence, it follows

$$
\Phi_{r}^{\prime}(f ; h)=\lim _{t \rightarrow 0^{+}} \frac{\Phi_{r}(f+t h)-\Phi_{r}(f)}{t}=2 \int_{D} h K f d x .
$$

Since $K f=u_{f} \in H^{1}(D)$, we have $\Phi_{r}^{\prime}(f)=2 u_{f}$, as desired.

Proof of Theorem 5.1. First, let us consider the following relaxed problem

$$
\inf _{f \in \overline{\mathcal{R}\left(f_{0}\right)}} \Phi_{r}(f) .
$$

Since $\overline{\mathcal{R}\left(f_{0}\right)}$ is weakly sequentially compact (Lemma 2.6(ii)) and $\Phi_{r}$ is weakly sequentially continuous (Lemma 5.2(i)), the problem (5.6) is solvable. Due to the convexity 
of $\overline{\mathcal{R}\left(f_{0}\right)}$ (Lemma 2.6(ii)) and strict convexity of $\Phi_{r}$ (Lemma 5.2(ii)), the solution to $(5.6)$ is unique and we denote it by $\hat{f}$. For an arbitrary $g \in \overline{\mathcal{R}\left(f_{0}\right)}$, we have $\hat{f}+t(g-\hat{f}) \in \overline{\mathcal{R}\left(f_{0}\right)}$ for all $t \in(0,1)$. So, by applying Lemma $5.2($ iii $)$, it follows that

$$
0 \leq \lim _{t \rightarrow 0^{+}} \frac{\Phi_{r}(\hat{f}+t(g-\hat{f}))-\Phi_{r}(\hat{f})}{t}=2 \int_{D} u_{\hat{f}}(g-\hat{f}) d x .
$$

This means

$$
\int_{D} u_{\hat{f}} g d x \geq \int_{D} u_{\hat{f}} \hat{f} d x \quad \forall g \in \overline{\mathcal{R}\left(f_{0}\right)} .
$$

Hence, $\hat{f}$ minimizes the linear functional $L(h)=\int_{D} u_{\hat{f}} h d x$ relative to $g \in \overline{\mathcal{R}\left(f_{0}\right)}$. On the other hand, from the differential equation in (5.1), in conjunction with [18, Lemma 7.7], it follows that the graph of $u_{\hat{f}}$ has no significant flat section on $S(\hat{f})$. By using Lemma 2.12, we infer the existence of a nonincreasing function $\eta$ such that $\eta\left(u_{\hat{f}}\right) \in \mathcal{R}\left(f_{0}\right)$. Applying Lemma 2.8, we must have $\hat{f}=\eta\left(u_{\hat{f}}\right) \in \mathcal{R}\left(f_{0}\right)$ as desired.

Our second result of this section is the following.

TheOREm 5.3. Let $D$ be a ball centered at the origin with radius a. Then, the problem (5.3) has a unique solution $\hat{f} \in \mathcal{R}\left(f_{0}\right)$. Moreover, $\hat{f}$ is radially nondecreasing and it satisfies $\hat{f}=\eta\left(u_{\hat{f}}\right)$ a.e. in $D$ for some nonincreasing function $\eta$. Furthermore, $u_{\hat{f}}$ is positive throughout $\bar{D}$.

Proof. In light of Theorem 5.1, the problem (5.3) has a unique solution $\hat{f} \in \mathcal{R}\left(f_{0}\right)$ such that $\hat{f}=\eta\left(u_{\hat{f}}\right)$ for some nonincreasing function $\eta$. Thus, $\hat{u} \equiv u_{\hat{f}}$ is a solution of the following boundary value problem

$$
\begin{cases}-\Delta u=\eta(u) & \text { in } D \\ \frac{\partial u}{\partial \nu}+\beta u=0 & \text { on } \partial D .\end{cases}
$$

Recalling that $\eta: \mathbb{R} \rightarrow \mathbb{R}^{+}$is nonincreasing, we consider $\eta(m)=M>0$ for some $m \in \mathbb{R}$. Then, set $\zeta(t)=-\int_{m}^{t} \eta(s) d s$. Since $\eta$ is nonincreasing, $\zeta$ is convex and clearly continuous. We introduce the energy functional related to $(5.7), J: H^{1}(D) \rightarrow \mathbb{R}$, by

$$
J(u)=\frac{1}{2} \int_{D}|\nabla u|^{2} d x+\frac{1}{2} \beta \int_{\partial D} u^{2} d \mathcal{H}^{N-1}+\int_{D} \zeta \circ u d x .
$$

By using the coercivity of $a(\cdot, \cdot)$, coupled with the fact that $\eta$ is nonincreasing and nonnegative, we derive

$$
\begin{aligned}
J(u) & \geq c\|u\|^{2}+\int_{\{u>m\}} \zeta \circ u d x \geq c\|u\|^{2}-M \int_{D}|u-m| d x \\
& \geq c\|u\|^{2}-C\|u\|-C .
\end{aligned}
$$

Thus, $J$ is coercive. ${ }^{6}$ On the other hand, we have

$$
\int_{D} \zeta \circ \hat{u} d x \leq \int_{\{\hat{u}<m\}} \zeta \circ \hat{u} d x \leq \int_{\{\hat{u}<m\}}(m-\hat{u}) \eta(\hat{u}) d x \leq\|m-\hat{u}\|_{2}\|\hat{f}\|_{2}<\infty .
$$

\footnotetext{
${ }^{6}$ For the coercivity of $J(\cdot)$, we mean $J(u) \rightarrow+\infty$ as $\|u\| \rightarrow+\infty$.
}

Copyright $@$ by SIAM. Unauthorized reproduction of this article is prohibited. 
From this, we infer $J(\hat{u})<\infty$, i.e., $J(\cdot)$ is proper in the sense of convex analysis; see for example [14]. Since $J$ is strictly convex, by applying the direct method of calculus of variations, we deduce $\hat{u}$ must be the unique minimizer of $J$.

Next, we fix an arbitrary unit vector $\vec{e}$. Since $\zeta$ is continuous, we can apply Lemma 2.13 to obtain

$$
\begin{aligned}
J(\hat{u}) & =\frac{1}{2} \int_{D}|\nabla \hat{u}|^{2} d x+\frac{1}{2} \beta \int_{\partial D} \hat{u}^{2} d \mathcal{H}^{N-1}+\int_{D} \zeta \circ \hat{u} d x \\
& \geq \frac{1}{2} \int_{D}\left|\nabla \hat{u}^{*}\right|^{2} d x+\frac{1}{2} \beta \int_{\partial D} \hat{u}^{*^{2}} d \mathcal{H}^{N-1}+\int_{D} \zeta \circ \hat{u}^{*} d x \geq J(\hat{u}) .
\end{aligned}
$$

As $\hat{u}$ is the unique minimizer of $J$, we must have $\hat{u}=\hat{u}^{*}$. Since the vector $\vec{e}$ is arbitrary, we deduce $\hat{u}$ is radial. By using the formula $\hat{f}=\eta(\hat{u})$, we infer $\hat{f}$ is radial as well.

At this stage, let us consider the following initial value problem

$$
-\frac{1}{r^{N-1}}\left(r^{N-1} u^{\prime}\right)^{\prime}=\hat{f}(r), \quad u^{\prime}(0)=0, \quad u^{\prime}(a)+\beta u(a)=0 .
$$

We know that $\hat{u}$ is the unique solution of (5.8). By integrating (5.8) from 0 to $r$, we derive

$$
r^{N-1} \hat{u}^{\prime}(r)=-\int_{0}^{r} s^{N-1} \hat{f}(s) d s .
$$

Thus, $\hat{u}^{\prime} \leq 0$, since $\hat{f} \geq 0$. Hence $\hat{u}$ is nonincreasing. By using the formula $\hat{f}=\eta(\hat{u})$ again, we see that $\hat{f}$ is radially nondecreasing as desired.

To finish the proof, we need to show $\hat{u}>0$. To this end, we first find an explicit formula of $\hat{u}$. Substituting $r=a$ in (5.9), we get

$$
\hat{u}^{\prime}(a)=-\frac{1}{a^{N-1}} \int_{0}^{a} s^{N-1} \hat{f}(s) d s .
$$

Using the boundary condition in (5.8), we deduce

$$
\hat{u}(a)=\frac{1}{\beta a^{N-1}} \int_{0}^{a} s^{N-1} \hat{f}(s) d s .
$$

Now, integrating (5.9) from $r$ to $a$ yields

$$
\begin{aligned}
\hat{u}(r) & =\hat{u}(a)+\int_{r}^{a} \frac{1}{t^{N-1}} \int_{0}^{t} s^{N-1} \hat{f}(s) d s d t \\
& =\frac{1}{\beta a^{N-1}} \int_{0}^{a} s^{N-1} \hat{f}(s) d s+\int_{r}^{a} \frac{1}{t^{N-1}} \int_{0}^{t} s^{N-1} \hat{f}(s) d s d t,
\end{aligned}
$$

where we have used (5.10) in the second equality. Clearly, $\hat{u}(r)>0$ for all $r \in[0, a]$. So, we have $\hat{u}>0$ on $\bar{D}$ as desired.

Remark 5.4. We could also prove Theorem 5.3 by using a rotation lemma as in the proof of [15, Theorem 3.2].

The following is an immediate consequence of Theorems 5.1 and 5.3.

Corollary 5.5. Let $E$ be a measurable subset of $D$ satisfying $0<|E|<|D|$, and let $f_{0}=\chi_{E}$. The problem (5.3) has a unique solution $\chi_{\hat{E}} \in \mathcal{R}\left(\chi_{E}\right)$, where $\hat{E}=\left\{u_{\chi_{\hat{E}}}<m\right\}$ for some positive $m$. If $D$ is a ball centered at the origin with radius a, then $\hat{E}$ is a ring around the boundary. 
The next main result of this section is a converse of Corollary 5.5.

TheOREm 5.6. Let $D$ be a bounded, connected domain in $\mathbb{R}^{N}(N \geq 2)$ with $C^{1,1}$ boundary, and consider a measurable set $E \subseteq D$ satisfying $0<|E|<|D|$. If the solution of (5.3) with $f_{0}=\chi_{E}$ is $\chi_{\hat{E}}$, in which $\hat{E}=\left\{u_{\chi_{\hat{E}}}<m\right\}$ for some $m>0$ and $\hat{E}^{c}$ is a ball centered at the origin, then $D$ is also a ball centered at the origin.

Proof. We divide the proof into two steps.

Step 1: $u_{\chi_{\hat{E}}}$ is radially nonincreasing on $\bar{D}$. This can be shown by similar technicalities as in the proof of Theorem 3.3 with test functions listed in Table 1.

Step 2: $D$ is necessarily a ball centered at the origin. We will use some ideas from the proof of [22, Theorem 2]. In order to derive a contradiction, let us suppose $D$ is not a ball centered at the origin. Since $D$ is bounded and $\hat{E}^{c}$ is a ball centered at the origin, there exist $B(0, a)$ and $B(0, b)$ satisfying $0<a<b<\infty$ such that $B(0, a)$ is the largest ball centered at the origin with $B(0, a) \subseteq D$, while $B(0, b)$ is the smallest one satisfying $D \subseteq B(0, b)$. Furthermore, there exists $x_{a}, x_{b} \in \partial D$ such that $\left|x_{a}\right|=a$ and $\left|x_{b}\right|=b$. It follows that the outward unit normals to $D$ at $x_{a}$ and $x_{b}$ are $x_{a} /\left|x_{a}\right|$ and $x_{b} /\left|x_{b}\right|$, respectively. As a result, $u_{\chi_{\hat{E}}}$ satisfies the initial value problem (5.8) at $x=x_{a}$ and $x=x_{b}$. By using (5.10) with $\hat{f}=\chi_{\hat{E}}$, we obtain

$$
\left\{\begin{array}{l}
u_{\chi_{\hat{E}}}(a)=\frac{a}{N \beta}-\frac{R^{N}}{N \beta a^{N-1}}, \\
u_{\chi_{\hat{E}}}(b)=\frac{b}{N \beta}-\frac{R^{N}}{N \beta b^{N-1}},
\end{array}\right.
$$

where $R=\sqrt[n]{\frac{|D|-|E|}{\omega_{N}}}$. It is obvious that $u_{\chi_{\hat{E}}}(a)<u_{\chi_{\hat{E}}}(b)$ which is a contradiction to the conclusion of Step 1. This completes the proof of the theorem.

It is well known that the Dirichlet boundary condition can be recovered by setting $\beta=\infty$ in (5.1). The following result, in which we approximate the Dirichlet problem by Robin problems, seems to be interesting.

TheOrem 5.7. Let $D$ be a bounded domain in $\mathbb{R}^{N}(N \geq 2)$ with $C^{1,1}$ boundary, and $f_{0} \in L_{+}^{2}(D)$. Let $f_{\infty} \in \mathcal{R}\left(f_{0}\right)$ be the unique solution of the minimization problem (4.1) and $u_{\infty} \in H_{0}^{1}(D)$ satisfies

$$
\begin{cases}-\Delta u_{\infty}=f_{\infty} & \text { in } D \\ u_{\infty}=0 & \text { on } \partial D\end{cases}
$$

Let $\beta>0, f_{\beta} \in \mathcal{R}\left(f_{0}\right)$ be the unique solution of the minimization problem (5.3) and $u_{\beta} \in H^{1}(D)$ satisfies

$$
\begin{cases}-\Delta u_{\beta}=f_{\beta} & \text { in } D \\ \frac{\partial u_{\beta}}{\partial \nu}+\beta u_{\beta}=0 & \text { on } \partial D\end{cases}
$$

Then, we have

$$
\lim _{\beta \rightarrow \infty}\left\|f_{\beta}-f_{\infty}\right\|_{2}=0, \quad \lim _{\beta \rightarrow \infty}\left\|u_{\beta}-u_{\infty}\right\|_{2}=0, \quad \lim _{\beta \rightarrow \infty} \Phi_{r}\left(f_{\beta}\right)=\Phi_{d}\left(f_{\infty}\right) .
$$

Remark 5.8. Through the proofs of [15, Theorem 3.1] and Theorem 5.1, we know $f_{\infty}$ and $f_{\beta}$ are also the unique minimizers of the following relaxed problems, respectively:

$$
\Phi_{d}\left(f_{\infty}\right)=\inf _{f \in \overline{\mathcal{R}}\left(f_{0}\right)} \Phi_{d}(f)
$$


and

$$
\Phi_{r}\left(f_{\beta}\right)=\inf _{f \in \overline{\mathcal{R}\left(f_{0}\right)}} \Phi_{r}(f)
$$

Proof of Theorem 5.7. From (5.12) and (5.2), we have

$$
\int_{D}\left|\nabla u_{\beta}\right|^{2} d x+\beta \int_{\partial D} u_{\beta}^{2} d \mathcal{H}^{N-1}=\int_{D} f_{\beta} u_{\beta} d x .
$$

By using the coercivity of $a(\cdot, \cdot)$ and Lemma 2.6(i), we infer

$$
c\left\|u_{\beta}\right\|^{2} \leq\left\|f_{\beta}\right\|_{2}\left\|u_{\beta}\right\|_{2} \leq\left\|f_{\beta}\right\|_{2}\left\|u_{\beta}\right\|=\left\|f_{0}\right\|_{2}\left\|u_{\beta}\right\| .
$$

So, $\left\{u_{\beta}\right\}_{\beta>0}$ is bounded in $H^{1}(D)$. Since $\left\{f_{\beta}\right\}_{\beta>0}$ is bounded in $L^{2}(D)$, by passing to a subsequence, we have

$$
\begin{aligned}
& u_{\beta_{n}} \rightarrow \tilde{u} \quad \text { in } \quad H^{1}(D), \\
& u_{\beta_{n}} \rightarrow \tilde{u} \quad \text { in } \quad L^{2}(D) \text { and } L^{2}(\partial D), \\
& f_{\beta_{n}} \rightarrow \tilde{f} \quad \text { in } \quad L^{2}(D),
\end{aligned}
$$

where $\beta_{n} \rightarrow \infty$ as $n \rightarrow \infty$. From (5.12) and (5.2), it follows that

$$
\int_{D} \nabla u_{\beta_{n}} \cdot \nabla \varphi d x+\beta_{n} \int_{\partial D} u_{\beta_{n}} \varphi d \mathcal{H}^{N-1}=\int_{D} f_{\beta_{n}} \varphi d x \quad \forall \varphi \in H^{1}(D) .
$$

Passing to the limit in the last equation, the convergences (5.16), (5.17), and (5.18) imply

$$
\int_{\partial D} \tilde{u} \varphi d \mathcal{H}^{N-1}=0 \quad \forall \varphi \in H^{1}(D) .
$$

From this, we deduce $\tilde{u}=0$ on $\partial D$, hence, $\tilde{u} \in H_{0}^{1}(D)$. On the other hand, we have

$$
\int_{D} \nabla u_{\beta_{n}} \cdot \nabla \varphi d x=\int_{D} f_{\beta_{n}} \varphi d x \quad \forall \varphi \in H_{0}^{1}(D) .
$$

Passing to the limit, we get

$$
\int_{D} \nabla \tilde{u} \cdot \nabla \varphi d x=\int_{D} \tilde{f} \varphi d x \quad \forall \varphi \in H_{0}^{1}(D) .
$$

Whence, $\tilde{u} \in H_{0}^{1}(D)$ solves

$$
\begin{cases}-\Delta \tilde{u}=\tilde{f} & \text { in } D \\ \tilde{u}=0 & \text { on } \partial D\end{cases}
$$

At this stage, we recall the minimization problem (4.1). Since $\tilde{f} \in \overline{\mathcal{R}\left(f_{0}\right)}$ by (5.18), it follows from (5.14) that

$$
\int_{D} f_{\infty} u_{\infty} d x=\Phi_{d}\left(f_{\infty}\right) \leq \Phi_{d}(\tilde{f})=\int_{D} \tilde{f} \tilde{u} d x .
$$

Copyright (C) by SIAM. Unauthorized reproduction of this article is prohibited. 
Next, we denote by $w_{\beta_{n}} \in H^{1}(D)$ the solution of the following boundary value problem:

$$
\begin{cases}-\Delta w_{\beta_{n}}=f_{\infty} & \text { in } D, \\ \frac{\partial w_{\beta_{n}}}{\partial \nu}+\beta_{n} w_{\beta_{n}}=0 & \text { on } \partial D .\end{cases}
$$

It follows from (5.2) that

$$
\int_{D}\left|\nabla w_{\beta_{n}}\right|^{2} d x+\beta_{n} \int_{\partial D} w_{\beta_{n}}^{2} d \mathcal{H}^{N-1}=\int_{D} f_{\infty} w_{\beta_{n}} d x .
$$

By using the coercivity of $a(\cdot, \cdot)$, we obtain

$$
c\left\|w_{\beta_{n}}\right\|^{2} \leq\left\|f_{\infty}\right\|_{2}\left\|w_{\beta_{n}}\right\|_{2} \leq\left\|f_{\infty}\right\|_{2}\left\|w_{\beta_{n}}\right\| .
$$

So, $\left\{w_{\beta_{n}}\right\}$ is bounded in $H^{1}(D)$. By passing to a subsequence, if necessary, still denoted $\left\{w_{\beta_{n}}\right\}$, we have

$$
\begin{aligned}
& w_{\beta_{n}} \rightarrow \bar{u} \quad \text { in } \quad H^{1}(D), \\
& w_{\beta_{n}} \rightarrow \bar{u} \text { in } L^{2}(D) \text { and } L^{2}(\partial D) .
\end{aligned}
$$

Applying (5.2) to (5.20), we infer

$$
\int_{D} \nabla w_{\beta_{n}} \cdot \nabla \varphi d x+\beta_{n} \int_{\partial D} w_{\beta_{n}} \varphi d \mathcal{H}^{N-1}=\int_{D} f_{\infty} \varphi d x \quad \forall \varphi \in H^{1}(D) .
$$

Passing $n$ to infinity, in conjunction with (5.21), we obtain

$$
\int_{\partial D} \bar{u} \varphi d \mathcal{H}^{N-1}=0 \quad \forall \varphi \in H^{1}(D) .
$$

Hence, $\bar{u}=0$ on $\partial D$ and $\bar{u} \in H_{0}^{1}(D)$. On the other hand, we have

$$
\int_{D} \nabla w_{\beta_{n}} \cdot \nabla \varphi d x=\int_{D} f_{\infty} \varphi d x \quad \forall \varphi \in H_{0}^{1}(D) .
$$

Let $n$ go to infinity, and we get

$$
\int_{D} \nabla \bar{u} \cdot \nabla \varphi d x=\int_{D} f_{\infty} \varphi d x \quad \forall \varphi \in H_{0}^{1}(D),
$$

i.e., $\bar{u} \in H_{0}^{1}(D)$ solves

$$
\begin{cases}-\Delta \bar{u}=f_{\infty} & \text { in } D \\ \bar{u}=0 & \text { on } \partial D\end{cases}
$$

Recalling (5.11), by uniqueness, we must have $\bar{u}=u_{\infty}$.

Returning to the minimization problem (5.3), reciting (5.20), we derive

$$
\int_{D} f_{\beta_{n}} u_{\beta_{n}} d x=\Phi_{r}\left(f_{\beta_{n}}\right) \leq \Phi_{r}\left(f_{\infty}\right)=\int_{D} f_{\infty} w_{\beta_{n}} d x
$$

Passing $n$ to infinity by applying (5.17), (5.18), and (5.21), we infer

$$
\Phi_{d}(\tilde{f})=\int_{D} \tilde{f} \tilde{u} d x \leq \int_{D} f_{\infty} \bar{u} d x=\int_{D} f_{\infty} u_{\infty} d x=\Phi_{d}\left(f_{\infty}\right) .
$$

Copyright $@$ by SIAM. Unauthorized reproduction of this article is prohibited. 
Finally, from the minimization problem (4.1), combined with (5.19) and (5.22), we must have $\tilde{f}=f_{\infty}$ and $\tilde{u}=u_{\infty}$. By using (5.17), (5.18), and Lemma 2.14, we infer

$$
\lim _{n \rightarrow \infty}\left\|f_{\beta_{n}}-f_{\infty}\right\|_{2}=0, \quad \lim _{n \rightarrow \infty}\left\|u_{\beta_{n}}-u_{\infty}\right\|_{2}=0, \quad \lim _{n \rightarrow \infty} \Phi_{r}\left(f_{\beta_{n}}\right)=\Phi_{d}\left(f_{\infty}\right) .
$$

Because the sequence has only one accumulation point, the assertion follows.

Appendix A. Two analysis results. In section 3, the following two basic results from analysis were utilized, and we present them here for completeness. The first one is a variant of the Leibniz rule; see [16].

Proposition A.1. Let $S \equiv[a, b]^{2} \subseteq \mathbb{R}^{2}$ with $a<b, f \in L^{1}(S), \alpha \in(a, b]$, and $F(x) \equiv \int_{\alpha}^{x} f(x, t) d t$ for $x \in[a, b]$. Suppose $f(x, \cdot)$ is left continuous on $(a, b)$ for all $x \in[a, b]$, and for each $\epsilon \in(0, \alpha), f(\cdot, t)$ is Lipschitz continuous on $[\epsilon, b]$ with a uniform Lipschitz constant $L_{\epsilon}$ for all $t \in[a, b]$. Then, $F$ is left differentiable for all $x \in(a, b)$ and

$$
F^{\prime}(x-)=-f(x, x)+\int_{\alpha}^{x} \frac{\partial f}{\partial x-}(x, t) d t .
$$

Proof. We fix an arbitrary $x \in(a, b)$. For sufficiently small $h>0$, we have

$$
\frac{F(x-h)-F(x)}{h}=\int_{\alpha}^{x-h} \frac{f(x-h, t)-f(x, t)}{h} d t+\frac{\int_{x}^{x-h} f(x, t) d t}{h} .
$$

Without loss of generality, we assume $\alpha \geq x$. Since $f(\cdot, t)$ is Lipschitz continuous on $[\epsilon, b]$ with $0<\epsilon<x$ for all $t \in[a, b]$, we infer

$$
\lim _{h \rightarrow 0^{+}} \frac{f(x-h, t)-f(x, t)}{h} \chi_{[x-h, \alpha]}(t)=\frac{\partial f}{\partial x-}(x, t) \chi_{[x, \alpha]}(t) \quad \forall t \in[a, b] \text { a.e. }
$$

Recalling that the Lipschitz constant for $f(\cdot, t)$ on $[\epsilon, b]$ is $L_{\epsilon}$ for all $t \in[a, b]$, it follows from the Lebesgue dominated convergence theorem that

$$
\lim _{h \rightarrow 0^{+}} \int_{\alpha}^{x-h} \frac{f(x-h, t)-f(x, t)}{h} d t=\int_{\alpha}^{x} \frac{\partial f}{\partial x-}(x, t) d t .
$$

On the other hand, by using the left continuity of $f(x, \cdot)$ on $(a, b)$ for all $x \in[a, b]$, we deduce

$$
\lim _{h \rightarrow 0^{+}} \frac{\int_{x}^{x-h} f(x, t) d t}{h}=-f(x, x)
$$

Therefore, from (A.2), (A.3), and (A.4), we have (A.1) as desired. As $x$ is arbitrary in $(a, b)$, the assertion follows.

Remark A.2. Note that if $f(\cdot, t)$ is Lipschitz continuous on $[a, b]$ with a uniform Lipschitz constant $L$ for all $t \in[a, b]$, we could let $\alpha=a$ in the above proposition.

Proposition A.3. Let $(a, b) \subseteq \mathbb{R}$ with $a<b$, and $f \in C[a, b]$. If $f$ is left differentiable at every $x \in(a, b)$ and $f^{\prime}(x-)<0$ for all $x \in(a, b)$, then $f$ is (strictly) increasing on $[a, b)$. 
Proof. We argue by contradiction and suppose there exist $\alpha, \beta \in[a, b)$ with $\alpha<\beta$ such that $f(\alpha) \geq f(\beta)$. Since $f^{\prime}(\beta-)<0$, we may further assume $f(\alpha)>f(\beta)$ by choosing a slightly small $\beta$ if necessary. Then, the set $E \equiv\{x \in[\alpha, b): f(x)=f(\beta)\}$ is not empty and we claim that $\alpha=\inf E$. In order to derive a contradiction, we assume $\inf E=\gamma$ and $\gamma \in(\alpha, b)$. From the continuity of $f$, we must have $f(\gamma)=f(\beta)$. Since $f^{\prime}(\gamma-)<0$, coupled with the continuity, we infer the existence of $k \in(\alpha, \gamma)$ such that $f(k)=f(\beta)$ which contradicts inf $E=\gamma$. So, we have $\alpha=\inf E$ as desired. By using the continuity of $f$ again, we have $f(\alpha)=f(\beta)$ which is obviously a contradiction. This completes the proof of the proposition.

Acknowledgment. The authors wish to express their gratitude to the anonymous referee for suggestions and comments which improved the presentation greatly.

\section{REFERENCES}

[1] D. Arcoya, J. I. Diaz, ANd L. Tello, S-shaped bifurcation branch in a quasilinear multivalued model arising in climatology, J. Differential Equations, 150 (1998), pp. 215-225.

[2] C. Atkinson and C. R. Champion, On some boundary value problems for the equation $\nabla$. $(F(|\nabla w|) \nabla w)=0$, R. Soc. Lond. Proc. Ser. A Math. Phys. Eng. Sci., 448 (1995), pp. 269279 .

[3] S. Axler, P. Bourdon, and W. Ramey, Harmonic Function Theory, 2nd ed., Grad. Texts in Math. 137, Springer, New York, 2001.

[4] H. Brezis, Functional Analysis, Sobolev Spaces and Partial Differential Equations, Springer, New York, 2011.

[5] F. BRock, Rearrangements and applications to symmetry problems in PDE, in Handbook of Differential Equations: Stationary Partial Differential Equations. Vol. IV, Elsevier, Amsterdam, 2007, pp. 1-60.

[6] J. E. Brothers and W. P. Ziemer, Minimal rearrangements of Sobolev functions, J. Reine Angew. Math., 384 (1988), pp. 153-179.

[7] G. R. Burton, Rearrangements of functions, maximization of convex functionals, and vortex rings, Math. Ann., 276 (1987), pp. 225-253.

[8] G. R. BuRTon, Variational problems on classes of rearrangements and multiple configurations for steady vortices, Ann. Inst. H. Poincaré Anal. Non Linéaire, 6 (1989), pp. 295-319.

[9] G. R. Burton And J. B. MCLeod, Maximisation and minimisation on classes of rearrangements, Proc. Roy. Soc. Edinburgh Sect. A, 119 (1991), pp. 287-300.

[10] F. Cuccu, B. Emamizadeh, and G. Porru, Nonlinear elastic membranes involving the pLaplacian operator, Electron. J. Differential Equations, 2006 (2006), pp. 1-10.

[11] M. C. Delfour And J.-P. ZolÉsio, Shapes and Geometries. Metrics, Analysis, Differential Calculus, and Optimization, 2nd ed., Adv. Des. Control 22, SIAM, Philadelphia, 2011.

[12] J. Denzler, Windows of given area with minimal heat diffusion, Trans. Amer. Math. Soc., 351 (1999), pp. 569-580.

[13] J. I. DíAz, Nonlinear Partial Differential Equations and Free Boundaries. Elliptic Equations Vol. I, Res. Notes Math. 106, Pitman, Boston, 1985.

[14] I. Ekeland and R. TÉmam, Convex Analysis and Variational Problems, Classics Appl. Math. 28, SIAM, Philadelphia, 1999.

[15] B. Emamizadeh And Y. LiU, Constrained and unconstrained rearrangement minimization problems related to the p-Laplace operator, Israel J. Math., 206 (2015), pp. 281-298.

[16] H. Flanders, Differentiation under the integral sign, Amer. Math. Monthly, 80 (1973), pp. 615627.

[17] L. E. Fraenkel, An Introduction to Maximum Principles and Symmetry in Elliptic Problems, Cambridge Tracts in Math. 128, Cambridge University Press, Cambridge, 2000.

[18] D. Gilbarg and N. S. Trudinger, Elliptic Partial Differential Equations of Second Order, 2nd ed., Classics Math. 224, Springer, Berlin, 2001.

[19] A. Henrot, How to prove symmetry in shape optimization problems?, Control Cybernet., 25 (1996), pp. 1001-1013.

[20] A. Henrot and M. Pierre, Variation et optimisation de formes. Une analyse géométrique, Math. Appl. 48 (Berlin), Springer, Berlin, 2005.

[21] S. Kesavan, Symmetrization and Applications, Ser. Analysis 3, World Scientific, Hackensack, NJ, 2006. 
[22] J. Lamboley, A. Laurain, G. Nadin, and Y. Privat, Properties of Optimizers of the Principal Eigenvalue with Indefinite Weight and Robin Conditions, Calc. Var. Partial Differential Equations, 55 (2016), pp. 55-144.

[23] Y. Liu, Optimization Problems in Partial Differential Equations, Ph.D. thesis, University of Liverpool, Liverpool, England, 2015.

[24] Y. Liu, B. Emamizadeh, and A. Farjudian, Optimization problems with fixed volume constraints and stability results related to rearrangement classes, J. Math. Anal. Appl., 443 (2016), pp. 1293-1310.

[25] Y. Liu and B. Emamizadeh, Rearrangement minimization problems with indefinite external forces, Nonlinear Anal., 145 (2016), pp. 162-175.

[26] H. L. Royden, Real Analysis, 3rd ed., Macmillan, New York, 1988.

[27] E. Sperner, JR., Spherical symmetrization and eigenvalue estimates, Math. Z., 176 (1981), pp. $75-86$.

[28] G. TALEnti, Gradient estimates, rearrangements and symmetries, in Differential Equations with Applications to Mathematical Physics, Math. Sci. Eng. 192, Academic Press, Boston, 1993, pp. 307-312.

Copyright $@$ by SIAM. Unauthorized reproduction of this article is prohibited. 